Journal of Low Temperature Physics manuscript No.

(will be inserted by the editor)

\section{Deployment of POLARBEAR-2A}

Daisuke Kaneko $^{a}$. S. Adachi ${ }^{b}$. P. A. R. Ade ${ }^{c}$.

M. Aguilar ${ }^{d}$. Y. Akiba ${ }^{e, f}$. K. Arnold ${ }^{g}$.

C. Baccigalupi ${ }^{h, i}$. D. Barron ${ }^{j}$. D. Beck ${ }^{k}$.

S. Beckman ${ }^{l}$. F. Bianchini ${ }^{m}$. D. Boettger ${ }^{n}$.

J. Borrill ${ }^{o, p} \cdot$ J. Carron ${ }^{q}$. S. Chapman ${ }^{r}$.

K. Cheung ${ }^{l} \cdot$ Y. Chinone ${ }^{a, l} \cdot$ K. Crowley ${ }^{g}$.

A. Cukierman ${ }^{s}$. M. Dobbs ${ }^{t}$. R. Dunner ${ }^{n}$.

H. El-Bouhargani ${ }^{k}$. T. Elleflot ${ }^{g}$. J. Errard $^{k}$.

G. Fabbian ${ }^{q}$. S. M. Feeney ${ }^{u}$. C. Feng ${ }^{v}$.

T. Fujino ${ }^{w} \cdot$ N. Galitzki ${ }^{g}$ - A. Gilbert ${ }^{t}$.

N. Goeckner-Wald ${ }^{l}$. J. Groh ${ }^{l}$. G. Hall ${ }^{x}$.

N. W. Halverson ${ }^{y, z}$. T. Hamada ${ }^{e, a a}$.

M. Hasegawa ${ }^{e, f} \cdot$ M. Hazumi ${ }^{a, e, f, a b}$. C. A. Hill ${ }^{l, a c}$.

L. Howe ${ }^{g} \cdot$ Y. Inoue ${ }^{a d} \cdot$ G. Jaehnig ${ }^{y, z}$. O. Jeong ${ }^{l}$.

N. Katayama ${ }^{a}$ - B. Keating ${ }^{g}$. R. Keskitalo ${ }^{o, p}$.

S. Kikuchi ${ }^{w}$. T. Kisner ${ }^{o, p}$. N. Krachmalnicoff ${ }^{h}$.

A. Kusaka ${ }^{a, a c, a e} \cdot$ A. T. Lee ${ }^{l, a c, a f}$. D. Leon ${ }^{g}$.

E. Linder ${ }^{o, a c} \cdot$ L. N. Lowry ${ }^{g}$ · A. Mangul,ac .

F. Matsuda ${ }^{a}$. Y. Minami ${ }^{e}$. M. Navaroli ${ }^{g}$.

H. Nishino ${ }^{a g}$. J. Peloton ${ }^{a h}$ - A. T. P. Pham $^{m}$.

D. Poletti ${ }^{h}$. G. Puglisi ${ }^{a i}$. C. L. Reichardt ${ }^{m}$.

C. Ross ${ }^{r}$. Y. Segawa ${ }^{e, f}$. M. Silva-Feaver ${ }^{g}$.

P. Siritanasak ${ }^{g}$ - N. Stebor ${ }^{a j}$. R. Stompor ${ }^{k}$.

A. Suzukiac ${ }^{a c}$ O. Tajima ${ }^{b} \cdot$ S. Takakura ${ }^{a}$.

S. Takatori ${ }^{e, f} \cdot$ D. Tanabe $e^{e, f} \cdot$ G. P. Teply ${ }^{g}$.

T. Tomaru ${ }^{a k} \cdot$ C. Tsai ${ }^{g} \cdot$ C. Verges ${ }^{k}$.

B. Westbrook ${ }^{a f}$. Y. Zhou ${ }^{l}$

the date of receipt and acceptance should be inserted later

${ }^{a}$ Kavli Institute for the Physics and Mathematics of the Universe (WPI), UTIAS, the University of Tokyo, Kashiwa, Chiba 277-8583, Japan . ${ }^{b}$ Department of Physics, Kyoto University $\cdot{ }^{c}$ School of Physics and Astronomy, Cardiff University $\cdot{ }^{d}$ Departamento de Física, FCFM, Universidad de Chile $\cdot{ }^{e}$ High Energy Accelerator Research Organization (KEK) $\cdot{ }^{f}$ The Graduate University for Advanced Studies (SOKENDAI) . ${ }^{g}$ Department of Physics, University of California, San Diego $\cdot{ }^{h}$ The International School for Advanced Studies (SISSA) $\cdot{ }^{i}$ The National Institute for Nuclear Physics (INFN) $\cdot{ }^{j}$ Department of Physics and Astronomy, University of New Mexico $\cdot{ }^{k}$ AstroParticule et Cosmologie, Université Paris Diderot $\cdot{ }^{l}$ Department of Physics, University of California, Berkeley ${ }^{m}$ School of Physics, University of Melbourne ${ }^{n}$ Centro de Astro-Ingeniería, Pontificia Universidad Católica de Chile ${ }^{\circ}$ Space Sciences Laboratory, University of California $\cdot{ }^{p}$ Computational Cosmology Center, Lawrence Berkeley National Laboratory, ${ }^{q}$ Department of Physics and Astronomy, University of Sussex ${ }^{r}$ Department of Physics and Atmospheric Science, Dalhousie University $\cdot{ }^{s}$ Kavli Institute for Particle Astrophysics and Cosmology, Stanford University $\cdot{ }^{t}$ Physics Department, McGill University . ${ }^{u}$ Center for Computational Astrophysics, Flatiron Institute $\cdot{ }^{v}$ Department of Physics, University of Illinois at Urbana-Champaign $\cdot{ }^{w}$ College of Engineering Scinence, Yokohama National University ${ }^{x}$ School of Physics and Astronomy, University of Minnesota $\cdot{ }^{y}$ Center for Astrophysics and Space Astronomy, University of Colorado ${ }^{z}$ Department of Physics, University of Colorado ${ }^{a} a$ Astronomical Institute, Graduate School of Science, Tohoku University . ${ }^{a b}$ Institute of Space and Astronautical Science, Japan Aerospace Exploration Agency . ${ }^{a c}$ Physics Division, Lawrence Berkeley National Laboratory ${ }^{a d}$ Department of Physics, National Central University . ${ }^{a e}$ Department of Physics, School of 
1 Abstract Polarbear-2A is the first of three receivers in the Simons Array, a cosmic microwave background experiment located on the Atacama Plateau in Chile. POLARBEAR2A was deployed and achieved the first light in January 2019 by mapping the microwave emission from planet observations. Commissioning work is underway to prepare the receiver for science observations.

6 Keywords CMB, $B$-mode polarization, Millimeter wave, TES bolometer

\section{Introduction}

\section{$8 \quad 1.1$ Motivations for B-mode studies}

Since its discovery in 1964, the cosmic microwave background (CMB) has been one of the most important sources of cosmological information. Many observations have been performed since then, and observation technology has also greatly advanced (see review in [1] and references therein). A front line is the observation of $B$-mode polarization, which is a pattern characterized by odd-parity in spatial inversion.

A goal of $B$-mode polarization observation is to provide evidence of a nonzero tensorto-scalar ratio, $r$. It is defined as the ratio of the amplitudes of the tensor and scalar perturbations in the early universe. Tensor perturbations are only caused by primordial gravitational waves that are generated by cosmic inflation [2,3]. Therefore, a nonzero $r$ would provide evidence of the inflation, and if discovered, the value of $r$ would help to distinguish between inflation models. Another motivation for observing $B$-mode is to measure the sum of neutrino masses $\Sigma_{i=1}^{3} m_{v}^{i}[4,5]$. The $B$-mode anisortopies at small scales arise from $E$-mode by the effect of gravitational lensing, which are sensitive to $\Sigma_{i=1}^{3} m_{v}^{i}$.

\subsection{Concept of POlarbear and Simons Array experiments}

The Polarbear (PB) experiment is designed to observe the CMB polarization and is mounted on the Huan Tran Telescope (HTT). With a primary mirror of $2.5 \mathrm{~m}$ effective diameter and with a continuously rotating half-wave plate (HWP) [6], PB achieved high sensitivity across a wide multipole range where both primordial gravitational waves and gravitational lensing $B$-modes are included. The original PB experiment began PB-1 observations in year 2012. Our results were published in papers [7-12].

The Simons Array (SA) is an upgrade of the PB experiment with three telescopes with newly designed receivers. Two additional telescopes of the same design ${ }^{1}$ have been built next to the original HTT. The new receivers are called Polarbear-2A (PB-2A), PoLARBEAR-2B (PB-2B), and POLARBEAR-2C (PB-2C), respectively by the order in which they are manufactured. The number of detectors per receiver is 7588: about six times more than that of PB-1. PB-2A and PB-2B are equipped with dichroic sensors to detect $90 \mathrm{GHz}$ and $150 \mathrm{GHz}$ bands. $\mathrm{PB}-2 \mathrm{C}$ also has dichroic sensors, but these are sensitive to frequency bands centered at 220 and $270 \mathrm{GHz}$ to detect thermal dust radiation. Because of additional frequency bands, SA will be able to more reliably separate the CMB signal from those produced by foreground sources.

Science, the University of Tokyo . ${ }^{a f}$ Radio Astronomy Laboratory, University of California, Berkeley . ${ }^{a g}$ Research Center for the Early Universe, School of Science, the University of Tokyo $\cdot{ }^{a h}$ Laboratoire de l'Accelerateur Lineaire $\cdot{ }^{a i}$ Department of Physics, Stanford University $\cdot{ }^{a j}$ Intel Corporation $\cdot{ }^{a k}$ National Astronomical Observatory of Japan, E-mail: daisuke.kaneko@ipmu.jp

\footnotetext{
1 The Nicholas Simons Telescope (North), and The Paul Simons Telescope (South)
} 
The expected sensitivities at SA are $\sigma(r)=0.006$ for $r=0.1$, and $\sigma\left(\Sigma_{i=1}^{3} m_{v}^{i}\right)=$ $40 \mathrm{meV}$ for the sum of neutrino masses. Both values are in $68 \%$ C.L., after 3 years of observation with expected performance, foreground reduced, and degeneracy mitigated with future optical surveys such as DESI [13].

\section{Design of POLARBEAR-2A}

A cross-sectional image of the PB-2A receiver is described in Fig. 1. The receiver can be separated into two sections: an "optics tube" and a "backend". CMB light enters the receiver through the window of the optics tube and finally focuses on the detector located at the backend.

\subsection{Optical system}

The HTT adopts a Gregorian-Dragone configuration [14] which can compensate for cross polarization made by each mirror. A receiver system is located around the Gregorian focus of the telescope. The POLARBEAR-2A receiver comprises a cryostat with three shells cooled by pulse tube refrigerators. The chambers inside are supported by insulating fiberglass laminate struts, and multilayer reflective insulators are installed to reduce thermal conduction. The outermost $300 \mathrm{~K}$ shell is also a vacuum chamber.

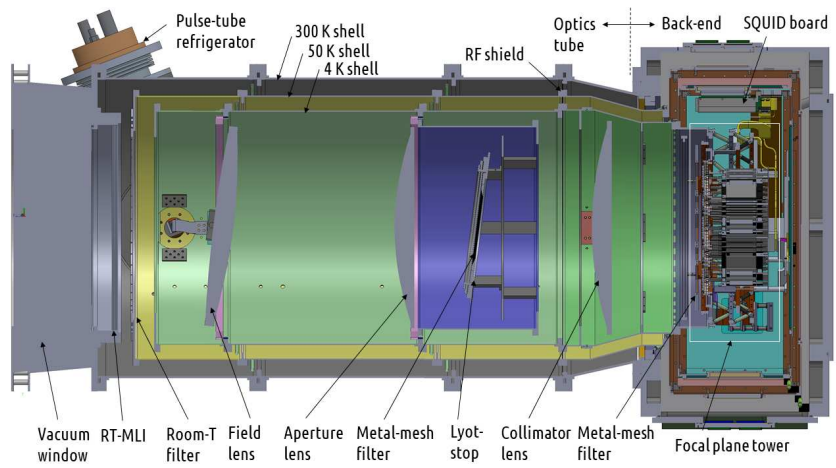

Fig. 1 Cross-sectional image of POLARBEAR-2A receiver. The criostat has $300 \mathrm{~K}, 50 \mathrm{~K}$, and 4 K shells. Light enters the receiver from foamed polypropylene window $(50 \mathrm{~cm}$ diameter, $20 \mathrm{~cm}$ thickness $)$ at the left side.

The optics tube contains a re-focusing optical system that consists of three alumina lenses. Details of fundamental optical design are found elsewhere [15]. To reduce reflections caused by the high refractive index (3.10) of alumina, two types of anti-reflection coatings are applied. A two-layered epoxy resin [16] is used for each curved face, and a sprayed mullite and porous-polyimide sheet [17] is used for each flat face. Radio transparent multi-layererd insulators (RT-MLIs [18]), an alumina filter and low-pass metal mesh filters are installed in the optical system to reduce the thermal load. The aperture (Lyot) stop is put inside the $4 \mathrm{~K}$ shell and tile-shaped epoxy based black body (KEK-black) [19] is mounted at its surface. The same black body absorber is applied to the inner surface of the $4 \mathrm{~K}$ shell. 
Fig. 2 shows the PB-2A detectors assembled in the focal plane tower (FPT). A 3-stage

\subsection{Readout system}

A schematic of the TES readout is shown in Fig. 4 [22]. PB-2A uses Digital Frequency-

Fig. 2 Assembled focal plane tower. Each with the seven hexagonal structure is a detector module, and the surface is covered with lenslets.

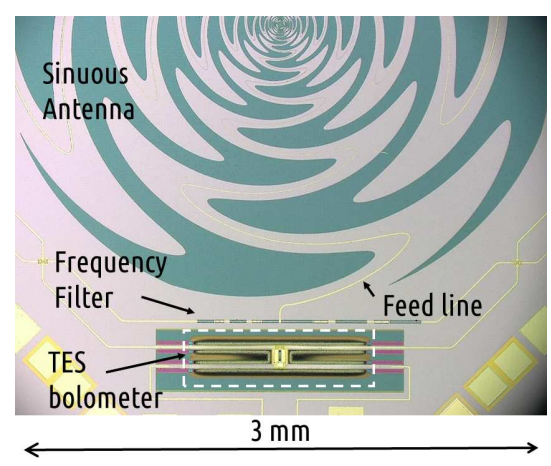

Fig. 3 Microscopic image of sinuous antenna and detector which are processed on a silicon wafer. The TES is located at the center of dashed rectangle.

The incident photons are coupled to an antenna by a silicon lenslet. Fig. 3 provides a zoomed-in photograph of a sinuous antenna. It is sensitive to two orthogonal linear polarizations and provides uniform response over a wide frequency range. A load resistor coupled with the antenna and the TES are on an "island", and heat from the load resister transmits to the TES. Division Multiplexing (DfMux) of factor 40 to reduce the thermal loading from outer electronics, and the total number of cables. In the DfMux method, TESs are coupled with LC resonators of different resonant frequencies and bias voltages are supplied in corresponding mixed frequencies. The multiplexed TES currents travel over low-inductance thermally insulating cables where they are transduced into a voltage by a SQUID array operating at 4 K. Input signal generation, output digitization, and network communication are controlled with "ICE boards" [23], FPGA-based electronics, installed in telescope comoving crates. The sampling frequency is $152 \mathrm{~Hz}$ for all channels. Data stored at the front-end computer is transferred down-site to the foot of the mountain by a direct radio-link, subsequently, transfered to servers located in the United States and Japan using the Internet. 


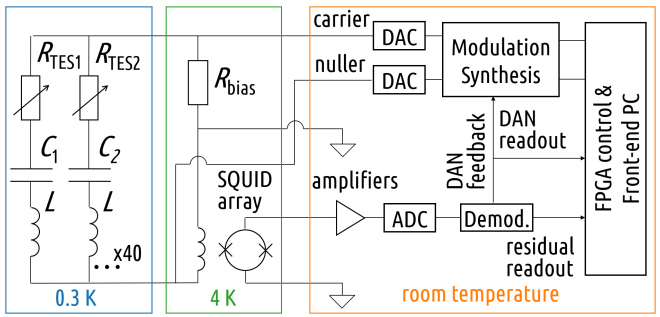

Fig. 4 Schematic diagram of PB-2A readout. Stages of different temperatures are thermally insulated with $\mathrm{NbTi}$ cable $(0.3 \mathrm{~K}-4 \mathrm{~K})$ and flexible wire harness (4 K - room Temp.).

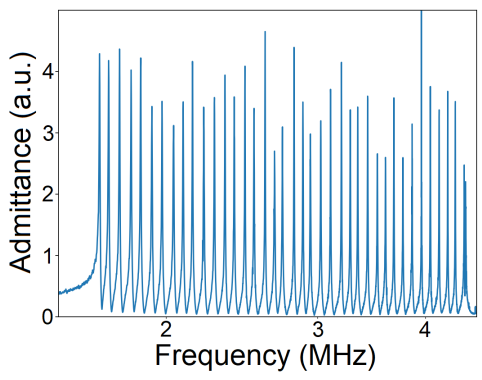

Fig. 540 different LC resonant peaks seen in laboratory test.

\section{Validation of POLARBEAR-2A}

\subsection{Laboratory testing}

Integration tests of the PB-2A receiver were conducted with a fully installed detector array and nearly all readout channels until the summer of 2018 at KEK, Japan. There are several fundamental functionalities that had to be confirmed before shipping to the site: (a) evacuation and cooling with refrigerators, (b) data acquisition tests with equivalent setup as the site and (c) optical performance at cryogenic temperature.

The evacuation and cooling were tested succsesfully at nominal operation temperature at $0.3 \mathrm{~K}$ for the detector stage. The typical hold time was 20 hours, which is long enough to operate in a 24 hour cycle. As this value is under a condition with additional neutral density filters for laboratory tests, the hold time should be a few hours longer without the filters at the site. Second, data acquisition was checked with full-scale TES channels under the same hardware configuration as that at the site [24]. Fig. 5 shows an example of the DfMux readout, in which forty peaks corresponding to multiplexed channels are clearly seen. The receiver noise was checked to ensure that the dominant noise was photon noise but not readout noise ( $\sim 20 \mathrm{pA} / \sqrt{\mathrm{Hz}}$ in normal conduction state). Third, the optical transmission of optics tube was confirmed. The optical efficiency from the window to the detector was measured with a liquid nitrogen vessel placed in front of the window. The optical efficiency was found to be $23 \%$ / $21 \%$ for $90 / 150 \mathrm{GHz}$ band, which is consistent with the expectation.

The spectral performance of the detector was examined with a Fourier transform spectrometer [25]. Some TESs of both bands are measured. Measured band centers were 85.2 / 143.9 GHz, band widths were $18.3 / 22.1 \mathrm{GHz}$ for $90 / 150 \mathrm{GHz}$ band channels, which were within the expected ranges. Spectroscopic tests for the entire detector array are planned at the site. The detectors were upgraded after these measurements. Detection efficiency is expected to be improved with upgraded detectors that use silicon nitride as the dielectric [21]. Operation of calibrators like a stimulator (described later) are also tested in the laboratory.

\subsection{Site commisioning}

In October 2018, the receiver was shipped to the Atacama Plateau. The assembly and installation onto the north telescope were completed in about one month and subsequently, site commissioning began. The cryogenic system was soon stabilized to enable test operation, then readout tests were successively performed. The achieved noise in the best case at the site is $\sim 15 \mathrm{pA} / \sqrt{\mathrm{Hz}}$ (in normal conduction state). The assembly and operation test of the HWP have been finished. 
Stimulator The stimulator is an artificial calibration source that uses black-body radiation from a ceramic heater (see Fig. 6). It is placed behind the secondary mirror, and signal is sent through a small hole in the mirror. In normal observations, stimulator data will be taken before and after each $\mathrm{CMB}$ scan and used for channel selection, relative gain calibration, and time-constant measurements. It is also used for many tests in deployment as a constant source. The signal was clearly seen with sufficient intensity by most $(>90 \%)$ of available detectors. Measured effective temperature is $45 / 70 \mathrm{mK}$ for $90 / 150 \mathrm{GHz}$ bands.

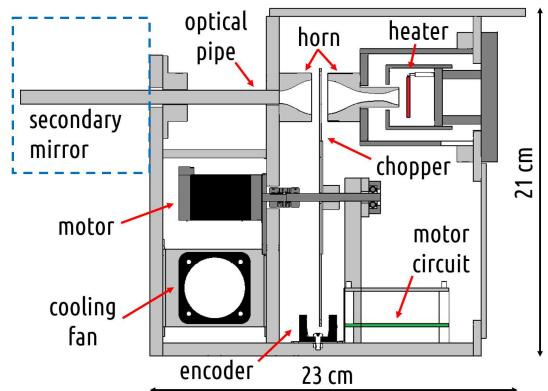

Fig. 6 Design of the stimulator for PB-2A. A ceramic heater ( $700 \mathrm{deg} \mathrm{C})$ is covered in total three layers of covers. The chopper modulates signal from 5 to $44 \mathrm{~Hz}$.

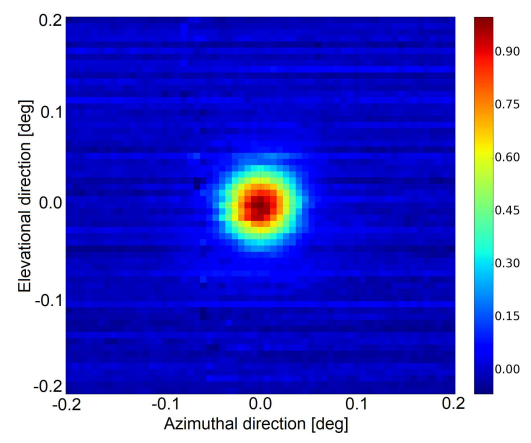

Fig. 7 Observed image of Venus averaged over $150 \mathrm{GHz}$ TESs in one detector module. This is a preliminary image before alignment of the receiver.

Planet observations Planet observations are useful for beam-shape characterization because planets are effectively point-like sources. We achieved the first light with an observation of Venus in January 2019. Fig. 7 is the first planet image which we observed with POLARBEAR-2A. Although the telescope had not yet been optimally aligned at this stage, data from several detectors during this scan showed circular images of Venus. Measured beam widths were 5.8 / 3.8 arcmin in FWHM for 90 / $150 \mathrm{GHz}$ channels, while design values are 5.0 / 3.5 arcmin. More recently, other planets such as Jupiter and Saturn, have been observed as well. These planet images are being used for alignment of the telescope and absolute scale calibration.

\section{Conclusion}

PB-2A, the first receiver for the Simons Array, is expected to have higher performance than the original PB-1, with about 6 times as many TES detectors as those of PB-1. Laboratory tests have finished and the PB-2A was deployed to the site in the Atacama Plateau. We achieved a successful operation of thousands of TESs and the first light with PB-2A was received in planet observations. The commissioning work continues to validate all the system to start CMB science observations.

Acknowledgements The Simons Array is supported by the Simons Foundation, the National Science Foundation (AST-1440338), the Moore Foundation, and the Heising-Simons Foundation. This work was supported by JSPS KAKENHI Grant Number JP26220709, JP15H05891, JP18H01240, JSPS Core-to-core Program, A.Advanced Research Networks., World Premier International Research Center Initiative (WPI), MEXT, Japan. 


\section{References}

1. R. Durrel, Classical and Quantum Gravity, 32(12), (2015), DOI: 10.1088/02649381/32/12/124007

2. U. Seljak and M. Zaldarriaga, Phys. Rev. Lett. 78, 2054 (1997)

3. M. Kamionkowski, A. Kosowsky, and A. Stebbins, Phys. Rev. Lett. 78, 2058 (1997)

4. D. J. Eisenstein, W. Hu, and M. Tegmark, Astrophys. J., 518, 2, 23 (1998)

5. M. Kaplinghat, L. Knox, and Y.-S. Song, Phys. Rev. Lett. 91, 241301 (2003)

6. C. A. Hill, et al, Proc. SPIE, 99142 U (2016), DOI: $10.1117 / 12.2232280$

7. P. A. R. Ade, et al. (PolarbearCollaboration), Phys. Rev. Lett., 112131302 (2014)

8. P. A. R. Ade, et al. (PolarbeArCollaboration), Astrophys. J., 794, 2, 171 (2014)

9. P. A. R. Ade, et al. (PolarbearCollaboration), Phys. Rev. Lett., 113, 021301 (2014)

10. J. Errard, et al. (PolarbearCollaboration), Astrophys. J., 809, 63 (2015)

11. P. A. R. Ade, et al. (PolarbearCollaboration), Phys. Rev. D, 92, 123509 (2015)

12. P. A. R. Ade, et al. (PolarbearCollaboration), Astrophys. J., 848, 121 (2017)

13. A. Aghamousa, et al. (The DESI Collaboration), arXiv 1611.00036 (astro-ph.IM), (2016)

14. C. Dragone, Bell Syst. Tech. J., 57, 7 (1978)

15. F. T. Matsuda, Doctoral Thesis, University of California San Diego, (2017)

16. D. Rosen, et al. Appl. Opt., 52, 33, (2013), DOI: 10.1364/AO.52.008102

17. Y. Inoue, et al. Appl. Opt., 55, 34, (2016), DOI: 10.1364/AO.55.000D22

18. J. Choi, et al. Rev. Sci. Instr., 84, 114502, (2013) DOI: 10.1063/1.4827081

19. Y. Inoue, Doctoral Thesis, Graduate University for Advanced Studies (SOKENDAI), (2016)

20. K. D. Irwin and G. C. Hilton, Cryogenic Particle Detection, C. Enss (Ed.), SpringerVerlag, (2005)

21. B. Westbrook, et al. J. Low Temp. Phys., 193, 758, (2018), DOI: 10.1007/s10909-0182059-0

22. K. Hattori, et al, Nucl. Instr. Meth. A, 732, 299-302, (2013), DOI: 10.1016/j.nima.2013.07.052

23. K. Bandura, et al, J. Astron. Instr. 05, 1641005, (2017)

24. D. Barron et al., Proc. SPIE, 1070808, (2018), DOI: $10.1117 / 12.2311502$

25. F. Matsuda, et al., Rev. Sci. Instr. 90, 115115 (2019), DOI: 10.1063/1.5095160, arXiv:1904.02901 (astro-ph.IM) 